The AstrophysiCAL Journal, 341 :L13-L16, 1989 June 1

(C) 1989. The American Astronomical Society. All rights reserved. Printed in U.S.A.

\title{
THE WARP OF THE GALACTIC STELLAR DISK DETECTED IN IRAS SOURCE COUNTS
}

\author{
S. DJorgovski ${ }^{1}$ AND CRAIg Sosin \\ Division of Physics, Mathematics, and Astronomy, California Institute of Technology \\ Received 1989 January 18; accepted 1989 March 13
}

\begin{abstract}
We investigate the tentative evidence for a warp in the Galactic stellar disk, similar to the well-known warp seen in the $\mathrm{H}$ I gas, using a sample of $\sim 90,000 I R A S$ point sources as disk tracers. The IRAS sources were selected on the basis of infrared colors, and most of them are probably evolved, dust-shell stars. Analysis of their distribution near the Galactic plane shows a systematic variation of characteristic latitude with the longitude. The sources are found preferentially above the plane near $l^{\mathrm{II}} \sim 90^{\circ}-100^{\circ}$, and below the plane near $l^{\mathrm{II}} \sim 240^{\circ}-270^{\circ}$, with the amplitude of the deviation increasing at the fainter flux levels (i.e., for more distant sources). Simple sine-wave models provide good fits to the data, whereas the null hypothesis (no variation with the longitude) can be rejected with a high degree of significance. This behavior is at least qualitatively as expected, if the IRAS sources follow the $\mathrm{H}$ I warp. We conclude that the stellar disk of our Galaxy is warped at large radii in a way similar to the $\mathrm{H}$ I layer, and that the warp is an important characteristic of our Galaxy as a whole. Since most of the IRAS stars are relatively old (several Gyr), we propose that the warp is a long-lasting phenomenon, possibly caused by asymmetries of the mass distribution in the outer regions of the Galactic dark halo. The projected amplitude of the IRAS warp may be lower than expected if the stars and the gas trace each other at all radii. We tentatively explain this as a signature of the radial truncation of the Galactic stellar disk. We also find that the mean disk plane, as delineated by the $I R A S$ sources, is $\sim 0.1-0.2$ below $b^{\text {II }}=0^{\circ}$; this may reflect the $z$-distance of the Sun above the disk plane.
\end{abstract}

Subject headings: galaxies: the Galaxy — infrared: general

\section{INTRODUCTION}

One of the principal features of the Galactic hydrogen disk is the warp at large galactocentric radii (Burke 1957; Kerr 1957; Westerhout 1957; Oort, Kerr, and Westerhout 1958). Good reviews of the warp and the morphology of the outer Galaxy gas layer are given, e.g., by Weaver $(1974 a, b)$, Henderson (1979), Kulkarni, Blitz, and Heiles (1982), Henderson, Jackson, and Kerr (1982), Burton and te Lintel Hekkert (1986), Burton and Deul (1987), and Burton (1988), who list other relevant references. Similar H I warps are often seen in other spiral galaxies, at radii where the optical isophotes are too faint to check whether their stellar disks are also warped (Sancisi 1976, 1983; van Woerden 1979). Innanen et al. (1982), following an early suggestion by Baade (1963) and Arp (1964), detected a low-surface brightness feature in M31, which they interpret as a signature of a warp of the stellar disk (cf. also Walterbos and Kennicutt 1987). Sandage and Humphreys (1980) claimed an evidence for the optical warp in M33. A somewhat less persuasive evidence for a warp in the stellar disk of NGC 4565 was published by van der Kruit and Searle (1981). Van der Kruit (1979) found a very marginal evidence for optical warps in three edge-on spirals, and his detection of a warp in NGC 5907 was subsequently confirmed in an extensive study by Sasaki (1987), who also lists many other relevant references. Theoretical models are reviewed, e.g., by Saar (1979), Toomre (1983), Sparke and Casertano (1988), and references therein.

To date, it is not clear whether the wrap is a persistent feature of the Galaxy, or a transient phenomenon. It is also unknown whether the Galactic stellar disk follows the $\mathrm{H}$ I warp. Optical studies of the young disk (Population I) tracers are severely hampered by the interstellar extinction and incom-

\footnotetext{
${ }^{1}$ Alfred P. Sloan Foundation fellow.
}

pleteness. Fernie (1968) detected a systematic longitudinal deviation in the mean Galactic latitude of Cepheids, and Graham (1970) and Garmany (1986) found a similar trend in distribution of $\mathrm{OB}$ stars, in the same general way as the $\mathrm{H}$ I layer. Fich and Blitz (1983) found evidence for the warp in the distribution of $\mathbf{H}$ II region in the outer Galaxy. Miyamoto, Yoshizawa, and Suzuki (1988) even tried to address the kinematics of the putative warp in OB stars. However, the incompleteness of the catalogs used in these studies makes it hard to estimate the significance of their results. Also, all of the optical Population I tracers used in these studies are young, with the ages generally smaller than a single rotation period of the disk, and could have formed in situ from the warped $\mathrm{H}$ I layer. They thus do not answer the question of how persistent is the warp. In this Letter, we report the discovery of a feature in IRAS point source counts, which we interpret as a signature of the warp in the Galactic old stellar disk.

\section{THE IRAS DATA SETS AND ANALYSIS}

The IRAS satellite (Neugebauer et al. 1984) opened a new, unobscured window for studies of Galactic structure. An example of an effective use of IRAS point source is the study of the Galactic bulge by Habing et al. (1985). Other work is presented and reviewed by Habing $(1986,1987 a, b, 1988)$, Chester (1986), or Beichman (1987). The principal advantages of the IRAS sample are insensitivity to the interstellar extinction, uniformity in selection, and completeness in the sky coverage.

Most IRAS sources identified with stars are asymptotic giant branch (AGB) or post-AGB stars with dust shells, including many Miras and LPVs (Chester 1986; Glass 1986, 1988; Habing 1986, 1987a, b, 1988; Herman, Burger, and Pennix 1986; Whitelock, Feast, and Catchpole 1986). These sources are luminous, with typical luminosities in the range $L_{\mathrm{IR}} \sim 2000-4000 L_{\odot}$ (Habing 1988), although the faint end of 
their luminosity function is still poorly known. They are easily detected by IRAS at the distance of the Galactic center-or of the warp $(\sim 10-20 \mathrm{kpc})$. Habing et al. (1985) suggest the following approximate formula for IRAS stellar sources:

$$
L_{\mathrm{IR}} \simeq 1.5 v f_{v}\left(4 \pi D^{2}\right) .
$$

Thus, a source with the flux of $0.5 \mathrm{Jy}$ in the IRAS $12 \mu \mathrm{m}$ band corresponds to an $L_{\mathrm{IR}} \simeq 2350 L_{\odot}$ source ( $\sim$ a typical shell star?) at the distance of $20 \mathrm{kpc}$, where the warp is prominent.

As described by those authors, it is possible to use IRAS colors to select stars and probable stars from the IRAS Point Source Catalog (PSC). Based on the information provided in these papers, we decided on the following selection criteria for stars or probable stars in the PSC: $F_{12} / F_{25} \geq 0.5, F_{12} / F_{60} \geq \frac{1}{3}$ (if detected at $60 \mu \mathrm{m}$ ), $F_{25} / F_{60} \geq 0.5$ (if detected at $25 \mu \mathrm{m}$ and $60 \mu \mathrm{m})$, where $F_{x}$ is the flux in the IRAS $x-\mu \mathrm{m}$ band. Furthermore, we restricted our study to the low latitudes, $\left|b^{\mathrm{II}}\right| \leq 10^{\circ}$. Two regions of the Galactic plane are affected by the incompleteness in the IRAS sky coverage: we skip the longitude ranges $78.5-91^{\circ}$ and $262^{\circ}-269^{\circ}$. Thus, the $80^{\circ}-90^{\circ}$ longitude bin disappears. There are enough sources left in the other affected bins, that our analysis is not compromised. This "basic" sample consists of 89,886 sources. Since we wanted to examine the global distribution of the sample in many longitude bins, and look at the relatively distant, and thus faint stars, we chose not to discriminate against the sources with poorer quality measurements: mere detections are enough for our purpose, and the precise fluxes are not really essential. We also subdivided the basic sample on the basis of the $12 \mu \mathrm{m}$ flux, $F_{12} / F_{25}$ infrared color, and Galactic latitude.

We first verified our conversion of the $(\alpha, \delta)$ coordinates from the PSC into the new Galactic $\left(l^{\mathrm{II}}, b^{\mathrm{II}}\right)$ system (cf. Lane 1979). We also established that the numerical errors of the conversion are unimportant, typically $\sim 5 \times 10^{-6}$ degrees.

For each of the samples, we divide the sources in $\Delta l^{\mathrm{II}}=10^{\circ}$ bins, and compute the median and the mean latitude $\left(\left\langle b^{\text {II }}\right\rangle\right)$ in each bin. A systematic variation of $\left\langle b^{\text {II }}\right\rangle$ with the longitude is evident in the basic sample and all of its subsamples, always in the sense that there are more sources above the mean plane in the $l^{\mathrm{II}} \sim 90^{\circ}-100^{\circ}$ region, and more sources below the mean plane in the $l^{\mathrm{II}} \sim 240^{\circ}-270^{\circ}$ region, which is at least qualitatively just as the behavior of the $\mathrm{H}$ I warp. The mean and the median latitudes in bins behave in the same way. Some examples are shown in Figure 1.

We computed the $\chi^{2}$ per degree of freedom $\left(\chi^{2} /\right.$ dof $)$ for the mean bin counts, assuming two models for the data. As the null hypothesis, we propose that the mean latitude should be constant, but not necessarily zero. As a simple model for the warp, we fit the counts as a sine wave of the form:

$$
\left\langle b^{\text {II }}\right\rangle=A \sin \left(l_{\text {bin }}^{\text {II }}-C\right)-B,
$$

where $l_{\text {bin }}^{\mathrm{II}}$ is the central longitude in each bin. The coefficients $A, B$, and $C$, and the $\chi^{2} /$ dof values are listed in Table 1 . The error-bars shown are the formal statistical $1 \sigma$ errors from the fits. A better model would assume some three-dimensional shape of the warp, include the projection effects, the luminosity function of the sources, etc., and would necessarily have many free parameters. In this preliminary report, we do only a very simple analysis of the data as described above, which is adequate for our purpose, and is easy to understand. It is clear from Table 1 that the null hypothesis is a very poor descriptor of the data, whereas the sine model is always better, and in general provides acceptable fits $\left(\chi^{2} /\right.$ dof $\left.\sim 1\right)$.

We also computed running medians of latitudes in groups of 41 sources as a function of longitude; the results appeared to be visually identical to the results from the binned data.

\section{DISCUSSION AND CONCLUSIONS}

The systematic variation in the $\left\langle b^{\text {II }}\right\rangle$ as a function of Galactic longitude is very similar to the projected distribution of the $\mathrm{H}$ I gas in the outer Galaxy. It persists in different samples of probable stellar sources selected from the IRAS PSC.

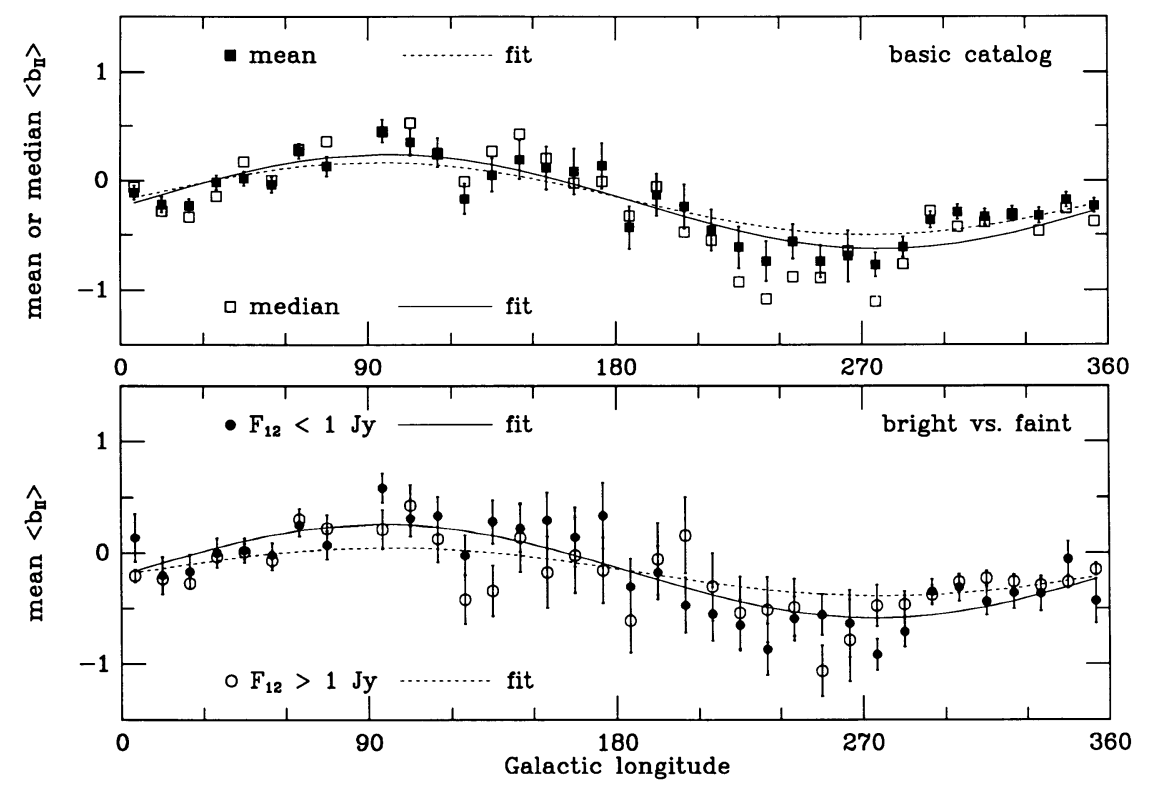

FIG. 1.-Variations of the mean Galactic latitude, $\left\langle b^{\mathrm{II}}\right\rangle$, as a function of Galactic longitude, in $10^{\circ}$ bins. The top panel shows the results for the basic catalog (see text): solid squares represent the mean latitudes in bins, and open squares the medians. The bottom panel divides the sources into $12 \mu \mathrm{m}$ flux: solid circles represent the fainter $(\sim$ more distant $)$ sources, and open circles represent the brighter $(\sim$ closer $)$ sources. The lines represent the corresponding sine model fits, as marked. 
TABLE 1

MODEL FITS FOR DIFFERENT SUBSAMPLES

\begin{tabular}{lrcrrrr}
\hline \hline Sample & $N_{\text {sources }}$ & $\mathrm{A}$ & $\mathrm{B}$ & $\mathrm{C}$ & $\begin{array}{c}\left(\chi^{2} / \text { dof }\right) \\
\text { model }\end{array}$ & $\begin{array}{c}\left(\chi^{2} / \text { dof }\right) \\
\text { null }\end{array}$ \\
\hline Basic (see text) & 89886 & $0.33 \pm 0.02$ & $0.17 \pm 0.02$ & $4 \pm 5$ & 2.17 & 7.93 \\
Faint $F_{12}<1 \mathrm{Jy}$ & 45249 & $0.42 \pm 0.04$ & $0.16 \pm 0.03$ & $5 \pm 6$ & 1.35 & 5.44 \\
$\ldots F_{12}<0.5 \mathrm{Jy}$ & 17627 & $0.61 \pm 0.06$ & $0.21 \pm 0.05$ & $-1 \pm 8$ & 1.59 & 4.58 \\
$\ldots$ 0.5 Jy $<F_{12}<1 \mathrm{Jy}$ & 27010 & $0.30 \pm 0.04$ & $0.12 \pm 0.04$ & $17 \pm 11$ & 1.01 & 2.51 \\
$\ldots$ 1 Jy $<F_{12}<2 \mathrm{Jy}$ & 20231 & $0.23 \pm 0.04$ & $0.14 \pm 0.05$ & $16 \pm 15$ & 0.96 & 1.73 \\
$\ldots F_{12}>2 \mathrm{Jy}$ & 5018 & $0.20 \pm 0.04$ & $0.19 \pm 0.04$ & $4 \pm 15$ & 2.84 & 3.71 \\
Bright $F_{12}>1 \mathrm{Jy}$ & 44637 & $0.22 \pm 0.03$ & $0.17 \pm 0.03$ & $7 \pm 8$ & 1.95 & 3.76 \\
Hot $F_{12}>F_{25}$ & 79963 & $0.34 \pm 0.03$ & $0.18 \pm 0.02$ & $2 \pm 5$ & 1.58 & 6.51 \\
Cold $F_{12}<F_{25}$ & 9923 & $0.36 \pm 0.03$ & $-0.04 \pm 0.04$ & $41 \pm 8$ & 10.3 & 13.1 \\
Plane $\left|b_{I I}\right|<2^{\circ}$ & 28903 & $0.04 \pm 0.01$ & $0.01 \pm 0.02$ & $19 \pm 21$ & 1.10 & 1.28 \\
\hline
\end{tabular}

The mean Galactic plane defined by this sample is on the average $0.1-0.15$ below the plane defined by the IAU $\left(l^{\mathrm{II}}, b^{\mathrm{II}}\right)$ system. The cause of this deviation is unclear. We verified that it is not an artifact of our coordinate conversions. It may be partly caused by the fact that the Sun is above the Galactic disk plane (the $z$-height of $\sim 15$ pc corresponds to $\sim 0.1$ deviation at the distance of $8 \mathrm{kpc}$ ). It may also reflect the imperfect match of the IAU $\left(l^{\mathrm{II}}, b^{\mathrm{II}}\right)$ system to the true physical disk plane. The $\left(l^{\text {II }}, b^{\text {II }}\right)$ system was defined for convenience with some roundoff in $(\alpha, \delta)$, the coordinates of the Galactic pole and inclination of the Galactic equator, and the uncertainty in the position of the pole is of the order $\sim 0.1$ (Blaauw et al. 1960; Gum, Kerr, and Westerhout 1960). This finding illustrates the potential of the IRAS data base in determining more accurately the position of the Galactic plane on the sky, and the $z$-height of the Sun, but such considerations are beyond the scope of this Letter and will be explored elsewhere.

The longitudes of the maximum warp are not affected significantly by the IRAS detector hysteresis effects, which are important in the central bulge. We repeated the sine fits by omitting the four bins closest to the Galactic center, and the results did not change appreciably. Another possible effect is the Galactic plane "noise shadowing" (see the IRAS Explanatory Supplement, Chap. 8), which could cause incompleteness at faint flux levels. According to the IRAS Explanatory Supplement, this effect is not important for $12 \mu \mathrm{m}$ point sources. If the hysteresis or shadowing effects from the scan crossings of the plane were important, the observed deviation would have been stronger at smaller latitudes, which is the opposite from what is observed. We are unaware of any other instrumental artifacts which could make spurious warplike deviations in the $\left\langle b^{\text {II }}\right\rangle$, similar to those we detected, and we conclude that the effect is real.

The amplitude of the longitudinal variation of $\left\langle b^{\mathrm{II}}\right\rangle$ increases for the fainter, and thus on the average more distant sources, as it would be expected from a continuously warped disk. The sine model fit described above is a good descriptor of the data, and essentially always better than the null hypothesis. The only subsample for which this is not the case in the sample of "cold" $\left(F_{25}>F_{12}\right)$ sources, where both fits are very poor, and some fraction of which may be a confusion from the infrared cirrus, or background galaxies. The "plane" subsample $\left(\left|b^{\mathrm{II}}\right|<2^{\circ}\right)$ is about equally well described by the sine model and the null hypothesis. This is as expected, since the warp removes the sources from the mean plane in a roughly symmetric manner and places them at higher latitudes, where the asymmetry in the projected source counts should be more prominent.

All these effects argue that we are seeing the same phenomenon in both $\mathrm{H}$ I and IRAS IR light. A large majority or all of the color-selected PSC sources are almost certainly luminous dust-shell stars, whose fluxes would place them roughly at the distances of the $\mathrm{H}$ I warp. We thus conclude that we are detecting the warp of the Galactic stellar disk, traced by the population of evolved stars. The warp is therefore a feature of our Galaxy as a whole, and not only of its $\mathrm{H}$ i gas layer.

Some important differences may be present, however. First, the amplitude of the IRAS warp is several times smaller than what would be expected of the sources following the $\mathrm{H}$ I warp exactly, at the distance of $10 \mathrm{kpc}$ or more. Undoubtedly, a part of this discrepancy is due to the projection effects: we integrate surface density of the sources through the whole disk, and our average luminosity estimates provide only a very crude distance indicator. Our source counts may be dominated by intrinsically fainter sources at distances of $\leq 10 \mathrm{kpc}$. Until the faint end of the luminosity function of IRAS stars is better determined, the exact geometry of the IRAS warp will remain uncertain. A similar discrepancy between the optical and $\mathrm{H} \mathrm{I}$ warps in NGC 5907 was found by Sasaki (1987). As proposed by Sasaki, this may be due to a cutoff in the stellar disk, which may be present in many or all disk galaxies (van der Kruit 1979; van der Kruit and Searle 1981), even if both the stellar and the $\mathrm{H}$ I warps follow the same curved plane. The $\mathrm{H}$ I disks generally extend further than the visible stellar disks (Sancisi 1983), and their warp amplitudes increase with the galactocentric radius. Thus, one may be able to constrain the radial extent of the Galactic old stellar disk, using the $I R A S$ source counts, but such investigation is beyond the scope of this Letter. An alternative hypothesis is that the two warps do not follow the same warped surface, but we consider that option to be less likely.

Another difference here is that whereas the Galactic $\mathrm{H}_{\text {I }}$ warp has a stronger amplitude in its northern side $\left(l^{\mathrm{II}} \sim 90^{\circ}\right.$ zone), the IRAS warp appears to be fairly symmetric. That, too, can be accomodated in the context of the truncated stellar disk hypothesis. Whereas it is reassuring that the stellar disk shows a symmetric behavior, we cannot answer why is the Galactic $\mathrm{H}$ I disk asymmetric.

Another important result is that the warp is traced by a relatively old stellar population. A younger population could have formed in situ from the warped gas disk, on time scales much shorter than the rotation period of the disk. However, if 
the warp is traced by an old stellar population, it would have to be a long-lived phenomenon. The typical ages of Mira variables and LPVs are estimated to be in the range 4-17 Gyr (Feast 1986), and the AGB stars in this range of luminosities are at least a few Gyr old (Aaronson and Mould 1985). This would argue against the mechanisms which explain the warps as a sporadic or transient event, due, e.g., to galaxy interactions.

Probably the most promising dynamical explanation for warps in galactic disks is that they are caused by aspherical, massive dark halos: a triaxial or an oblate halo, misaligned with a galactic disk, could cause the warping (Binney 1978, 1981; Petrou 1980; Toomre 1983; Dekel and Shlosman 1983; Sparke 1984a, $b$; Sparke and Casertano 1988). A generic expectation in many modern models of galaxy formation is that the halos should be triaxial, or at least oblate, product of early mergers (Frenk et al. 1988; Zurek, Quinn, and Salmon 1988).
The warps would then be common and persistent features of galactic disks, and manifest in their old stellar populations. However, as shown by Sparke and Casertano (1988), the oblate (axisymmetric) halos are sufficient, or even preferable; the halos which cause warps need not be triaxial. Further studies of the Galactic warp may thus constrain the shape and other parameters of the Galactic dark halo.

It is a great pleasure to thank Harold Weaver for inspiration in the early phases of this project. We are also indebted to Ivan King, Tom Soifer, and Nick Scoville for useful comments, and to the referee, Linda Sparke, who helped us in clarifying some important points. S. D. acknowledges a partial support from the NASA IRAS Astrophysics Data Program, from California Institute of Technology, and from Alfred P. Sloan Foundation. C. S. was supported by the Summer Undergraduate Research Fellowship at Caltech.
Aaronson, M., and Mould, J. 1985, Ap. J., 288, 551.

Arp, H. 1964, Ap. J., 139, 1045.

Baade, W. 1963, Evolution of Stars and Galaxies (Cambridge, Mass.: Harvard University Press), p. 73.

Beichman, C. 1987, Ann. Rev. Astr. Ap., 25, 521

Binney, J. 1978, M.N.R.A.S., 183, 779.

. 1981, M.N.R.A.S., 196, 455.

Blaauw, A., Gum, C. S., Pawsey, J. L., and Westerhout, G. 1960, M.N.R.A.S., 121, 123.

Burke, B. F. 1957, A.J., 62, 90

Burton, W. B. 1988, in Galactic and Extragalactic Radio Astronomy, ed. G.

Verschuur and K. Kellermann (New York: Springer), p. 295.

Burton, B. F., and Deul, E. R. 1987, in The Galaxy, ed. G. Gilmore and B. Carswell (Dordrecht: Reidel), p. 55.

Burton, B. F., and te Lintel Hekkert, P. 1986, Astr. Ap. Suppl., 65, 427.

Chester, T. 1986, in Light on Dark Matter, ed. F. Israel (Dordrecht: Reidel) p. 3 .
Dekel,

Dekel, A., and Shlosman, I. 1983, in IAU Symposium 100, Internal Kinematics and Dynamics of Galaxies, ed. E. Athanasoula (Dordrecht: Reidel), p. 187

Feast, M. W. 1986, in Light on Dark Matter, ed. F. Israel (Dordrecht: Reidel), p. 339.

Fernie, J. D. 1968, A.J., 73, 995 .

Fich, M., and Blitz, L. 1983, in Kinematics, Dynamics, and Structure of the Milky Way, ed. W. Shuter (Dordrecht: Reidel), p. 151.

Frenk, C. S., White, S. D. M., Davis, M., and Efstathiou, G. 1988, Ap. J., 327, 507.

Garmany, C. 1986, in Luminous Stars and Associations in Galaxies, ed. C. de Loore et al. (Dordrecht: Reidel), p. 19.

Glass, I. S. 1986, M.N.R.A.S., 221, 879 1988, M.N.R.A.S., 234, 115.

Graham, J. 1970, A.J., 70, 703 .

Gum, C. S., Kerr, F. J., and Westerhout, G. 1960, M.N.R.A.S. 121, 132.

Habing, H. J. 1986, in Light on Dark Matter, ed. F. Israel (Dordrecht: Reidel), p. 329.

. 1987a, in The Galaxy, ed. G. Gilmore and B. Carswell (Dordrecht: Reidel), p. 173.

. 1978b, in Comets to Cosmology, ed. A. Lawrence (Berlin: Springer) p. 81 .

1988, Astr. Ap., 200, 40.

\section{EFERENCES}

Habing, H. J., Olnon, F. M., Chester, T., Gillett, F., Rowan-Robinson, M., and Neugebauer, G. 1985, Astr. Ap., 152, L1.

Henderson, A. P. 1979, in IAU Symposium 89, The Large-Scale Structure of the Galaxy, ed. W. Burton (Dordrecht: Reidel), p. 493.

Henderson, A. P., Jackson, P. D., and Kerr, F. J. 1982, Ap. J., 263, 116.

Herman, J., Burger, J. H., and Pennix, W. H. 1986, Astr. Ap., 167, 247.

Innanen, K. A., Kamper, K. W., Papp, K. A., and van den Bergh, S. 1982, Ap. J., 254, 515 .

Kerr, F. J. 1957, A.J., $62,93$.

Kulkarni, S. R., Blitz, L., and Heiles, C. 1982, Ap. J. (Letters), 259, L63.

Lane, A. P. 1979, Pub. A.S.P., 91, 405

Miyamoto, M., Yoshizawa, M., and Suzuki, S. 1988, Astr. Ap., 194, 107.

Neugebauer, G., et al. 1984, Ap. J. (Letters), 278, L1

Oort, J. H., Kerr, F. J., and Westerhout, G. 1958, M.N.R.A.S., 118, 379.

Petrou, M. 1980, M.N.R.A.S., 191, 767.

Saar, E. 1979, in IAU Symposium 84, The Large-Scale Structure of the Galaxy, ed. W. Burton (Dordrecht: Reidel), p. 513.

Sancisi, R. 1976, Astr. Ap., 53, 159.

. 1983, in IAU Symposium 100, Internal Kinematics and Dynamics of Galaxies, ed. E. Athanasoula (Dordrecht: Reidel), p. 55.

Sandage, A., and Humphreys, R. 1980, Ap. J. (Letters), 236, L1.

Sasaki, T. 1987, Pub. Astr. Soc. Japan, 39, 849.

Sparke, L. S. $1984 a, A p . J ., 280,117$. 1984b, M.N.R.A.S., 211, 911 .

Sparke, L. S., and Casertano, S. 1988, M.N.R.A.S., 234, 873.

Toomre, A. 1983, in IAU Symposium 100, Internal Kinematics and Dynamics of Galaxies, ed. E. Athanasoula (Dordrecht: Reidel), p. 177.

van der Kruit, P. C. 1979, Astr. Ap. Suppl., 38, 15.

van der Kruit, P. C., and Searle, L. 1981, Astr. Ap., 95, 157.

van Woerden, H. 1979, in IAU Symposium 84, The Large-Scale Structure of the Galaxy, ed. W. Burton (Dordrecht: Reidel), p. 501.

Walterbos, R. A. M., and Kennicutt, R. C. 1987, Astr. Ap. Suppl., 69, 311.

Weaver, H. 1974a, in Highlights Astr., 3, 423.

. 1974b, in IAU Symposium 60, Galactic Radio Astronomy, ed. F. J. Kerr and S. C. Simonson III (Dordrecht: Reidel), p. 573.

Westerhout, G. 1957, Bull. Astr. Inst. Netherlands, 13, 201

Whitelock, P., Feast, M., and Catchpole, R. 1986, M.N.R.A.S., 221, 1.

Zurek, W. H., Quinn, P. J., and Salmon, J. K. 1988, Ap. J., 330, 519. 